Recepción: 28 / 02 / 2017

Aceptación: 17 / 04 / 2018

Publicación: 08 / 05 / 2018

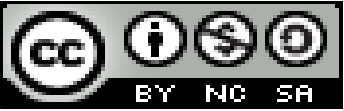

Ciencias de la salud

Artículo de Revisión

\title{
El parto en libre posición
}

Delivery in free position

Entrega em posição livre

\author{
Delia N. Crespo-Antepara ${ }^{\mathrm{I}}$ \\ deliacrespo57@hotmail.com
}

Correspondencia: deliacrespo57@ hotmail.com

I Magister en Gerencia Clínica en Salud Sexual y Reproductiva, Diplomado en Docencia Superior, Obstetriz, Docente en la Universidad de Guayaquil, Guayaquil, Ecuador. 


\section{Resumen}

En el presente trabajo, se propuso determinar las ventajas y desventajas del parto vertical. Al mismo tiempo, se consideró lo que históricamente ha sido en la mujer el parto en posición más o menos vertical, siendo las posiciones más utilizadas las de cuclillas, sentada o semisentada, la misma que fue considerada a partir del siglo XVI por el científico Mauriceau. La metodología fue de tipo descriptivo documental, cuya información se obtuvo mediante la revisión de 3486 historias clínicas del Hospital Materno Infantil Mariana de Jesús, entre mayo 2014 a febrero 2015. Entre sus resultados, se pudo determinar que el $15.81 \%$ tuvieron su parto en posición vertical. El $52.81 \%$ presento parto expulsivo cuya duración fue de menos de 5 minutos; el 63.88\% no presento ningún tipo de desgarro del canal blando; el 100\% de los recién nacidos obtuvieron en el Score de Apgar la puntuación de 8-9 al minuto; en lo referente al alumbramiento este tuvo una duración de 10 a 15 minutos en el $67.33 \%$ de los casos, existiendo en este parámetro, similitud con la que ocurre cuando el parto es en posición horizontal. Se pudo concluir, que la duración del parto expulsivo en la posición vertical fue más breve que el del parto horizontal.

Palabras clave: parto vertical; libre posición; parto horizontal; posiciones de parto.

\section{Abstract}

In the present work, it was proposed to determine the advantages and disadvantages of vertical delivery. At the same time, it was considered what historically has been in the woman delivery in more or less vertical position, being the most used positions squatting, sitting or semi-sitting, the same that was considered from the sixteenth century by the scientist Mauriceau. The methodology was documentary descriptive, whose information was obtained by reviewing 3486 medical records of the Mariana de Jesús Maternal and Child Hospital, between May 2014 and February 2015. Among its results, it was determined that $15.81 \%$ had their birth in position vertical. The $52.81 \%$ presented an expulsive delivery whose duration was less than 5 minutes; $63.88 \%$ did not present any type of tearing of the soft channel; $100 \%$ of the newborns obtained in the Apgar Score the score of 8-9 per minute; in relation to the delivery this lasted from 10 to 15 minutes in $67.33 \%$ of the cases, existing in this parameter, similarity to what occurs when the delivery is in horizontal position. It could be concluded that the duration of the expulsive delivery in the vertical position was shorter than that of the horizontal delivery. 
Keywords: vertical childbirth; free position; horizontal childbirth; delivery positions.

\section{Resumo}

No presente trabalho, foi proposto determinar as vantagens e desvantagens da entrega vertical. Ao mesmo tempo, foi considerado o que tem sido historicamente as mulheres o parto mais ou menos na vertical, sendo o de cócoras mais comumente usado, sentado ou semi-sitting, o mesmo que foi visto no século XVI pelas posições Mauriceau científicos. A metodologia foi memória descritiva cuja informação foi obtida por revisão dos prontuários de 3486 Maternidade Mariana de Jesus, de maio de 2014 a Fevereiro de 2015. Entre as suas conclusões, determinou-se que $15,81 \%$ deram à luz em posição vertical Os 52,81\% apresentaram parto expulsivo com duração inferior a 5 minutos; $63,88 \%$ não apresentaram nenhum tipo de ruptura do canal mole; $100 \%$ dos recém-nascidos obtiveram no Escore de Apgar a pontuação de 8-9 por minuto; em relação à entrega esta durou de 10 a 15 minutos em $67,33 \%$ dos casos, existindo neste parâmetro, semelhança com o que ocorre quando a entrega está na posição horizontal. Pode-se concluir que a duração do parto expulsivo na posição vertical foi menor que a do parto horizontal.

Palavras chave: parto vertical; posição livre; parto horizontal; posições de entrega.

\section{Introducción}

Entre las evidencias científicas sobre los beneficios del parto humanizado en vertical versus el parto horizontal, se pueden distinguir aspectos como la corriente creciente sobre el parto vertical en detrimento de la arraigada costumbre del parto horizontal, el mismo que se instauró para que los profesionales especialistas en la atención de parto, tengan más comodidad en su trabajo, se analiza las ventajas y desventajas de estas dos corrientes.

El parto humanizado, indica Pozzio (2014), habla sobre la integración de varios aspectos a tomar en cuenta, tales como el entorno, acompañamiento familiar, vestimenta de la parturienta, ingesta de bebidas, libre posición, ritual de la placenta, etc., al hacer un recorrido histórico, se encuentra como evidencia que las distintas posiciones adoptadas en el parto fueron (Engelman, 1997) analizadas en el año 1984, donde se discriminó y clasificó las diferentes posturas que adoptaban las mujeres de las distintas épocas, para observar los beneficios de unas sobre otras. Esa investigación generó un nuevo campo de estudio, donde varios investigadores continuaron 
analizando la evolución de las posiciones durante el expulsivo y la influencia que cada una de ellas tiene sobre la anatomía de la mujer, especialmente en el suelo perineal.

Walker (2008), en su libro "Cambio de la verticalidad a la horizontalidad para el parto", señala que además, la importancia que tiene tomar en cuenta la mala posición respecto de los riesgos maternos y fetales, los cuales pasan desapercibidos en la mujer, pero que suponen un problema grave de salud, tanto individual como colectivo, riesgos como desgarro de primero y segundo grado del periné, mayor sangrado y más consecuencias a tomar en cuenta, existe las condiciones del neonato evaluados por el test de APGAR.

En el parto vertical, Paniagua y Fernández (2013), que hoy se toma en cuenta más "la comodidad para los profesionales sanitarios más que para las propias madres" (p.24), menguando así los beneficios para la parturienta. Los beneficios naturales que gana una mujer en posición vertical, son entre otros: efectos respiratorios, mecánicos y de la gravedad, musculares, disminución de los riesgos de compresión de la aorta, eficiencia de la combinación matriz, disminución del trabajo del parto, menos dolor agudo en el expulsivo y postparto, amniorrexis espontánea más tardía, terminación espontánea, disminución de posibilidad de prolapso de cordón y mejora el estado anímico y psicológico de la mujer (Martínez y Col. 2001), de igual manera se presentan las desventajas: aumenta el riesgo de pérdida de sangre, más frecuencia de desgarros de poca profundidad, sobre todo en primíparas, puede resultar incómodo para los asistentes del parto, la posición en cuclillas algunas mujeres no la pueden soportar porque no tienen resistencia muscular, o porque tienen sobre peso. El parto horizontal (Carrillo, 2016) presenta las siguientes ventajas: menor riesgo de pérdida de sangre y facilita el movimiento de la mujer.

Existen recomendaciones (OMS, 1985) que advierten que no es recomendable colocar a la parturienta en posición dorsal, durante los períodos de dilatación y expulsivo, incluso estas recomendaciones van más allá, cada mujer debe decidir libremente la posición para realizar el expulsivo. Por lo que, en países como Colombia y Perú, la práctica del parto en posición vertical es muy frecuente.

En nuestro país, está práctica existió desde siempre, la misma que es evidenciada en estatuillas de cerámicas, que representan a la mujer en posición vertical, exhibida en el museo nacional de medicina "Eduardo Estrella” (Flores, 2007), está práctica se perdió al igual que en el resto del 
mundo, menos en las culturas de nuestros aborígenes. Por tratar de rescatar los saberes ancestrales puesto de manifiesto en nuestra constitución (Gobierno del Ecuador, 2008), el cual trata de devolver a nuestra cultura esta práctica, al considerar el reporte de 35 muertes maternas ocurridas en la provincia del Guayas en el mismo año, por complicaciones del embarazo, parto y puerperio, y por los reportes estadísticos realizados por la Encuesta Demográfica y de Salud Materna Infantil (ENDEMAIN, 2004), en que las mujeres campesinas preferían la atención tradicional del parto, el Ministerio de Salud Pública frente a estas realidades decide trabajar en el plan de reducción de muertes maternas dentro del Modelo de Atención Integral de Salud Familiar, Comunitaria e Intercultural, y capacitando al personal, e implementando áreas de atención en el parto vertical.

Actualmente en la Asamblea Nacional Ecuatoriana, se analiza y discute el proyecto del parto humanizado que entre otros componentes incorpora la libre posición de la mujer para parir, con la finalidad de reducir la razón de partos por cesárea, que en nuestro país supera la recomendada por la Organización Mundial de la Salud (OMS), siendo las provincias de Guayas y de Pichincha las que reportan las tasas más altas (EL UNIVERSO, 2016).

Si bien es cierto, que la posición vertical es la más fisiológica para el parto desde el punto de vista anatómico y biológico, seguimos con la cultura de hacer parir a la mujer en posición de litotomía por falta de información hacia la mujer sobre sus derechos a elegir la posición deseada; porque las instituciones de salud y los profesionales no están preparados para ofrecer este tipo de atención, y porque la mayoría de las mujeres consideran esta posición, propia de las culturas incivilizadas.

\section{Metodología}

La metodología fue de tipo descriptivo documental, que según señala Hernández, Fernández y Baptista (2010), está orientada a estudiar en forma intensiva las propiedades importantes de personas, grupos, comunidades o cualquier otro fenómeno sometido a análisis. En un estudio de esa naturaleza las variables de investigación se miden independientemente pero luego describir lo investigado. Por otro lado, la investigación es de tipo documental según Chávez (2007), implica un proceso de búsqueda, selección, lectura, registro, organización, descripción, análisis e interpretación de datos extraídos de fuentes documentales existentes en torno a un problema, con 
el fin de encontrar respuesta a interrogantes planteadas en cualquier área del conocimiento humano y desde esta perspectiva, el diseño fue no experimental.

Los datos del estudio, se obtuvieron mediante la revisión de 3486 historias clínicas del Hospital Materno Infantil Mariana de Jesús, entre mayo 2014 a febrero 2015.

\section{Resultado y Análisis}

Los resultados de la información obtenida posterior a la revisión de 3486 las historias clínicas de los partos atendidos entre mayo del 2014 a febrero del 2015 en la Maternidad Mariana de Jesús, 551 que representan el $15.81 \%$ tuvieron el parto en posición vertical, datos que concuerdan con los reportados en el estudio realizado durante 4 años en el hospital Raúl Maldonado de la ciudad de Quito.

En lo que respecta la duración del parto expulsivo, se estableció que el $52.81 \%$ de las parturientas duró menos de 5 minutos y en el $17.24 \%$ se prolongó por encima de 15 minutos tiempo que todavía sigue siendo menor al considerado en el parto horizontal; hallazgos diferentes reportó el estudio realizado en el hospital Vicente Morán del cantón Daule en el año 2013, en el cual cita que el $87 \%$ de los casos la duración del expulsivo fue menor a 5 minutos.

Los desgarros del canal blando del parto no se observaron en el $63.88 \%$ del total de parturientas, no así el $36.11 \%$ presentan desgarros de I hasta IV grado; probablemente se deba a la mala selección de las parturientas. Estos datos son discordados con los reportados con el estudio realizado en el Hospital Vicente Maldonado de la ciudad de Quito en el año 2013 en la cual la ausencia de desgarros fue del $88 \%$.

El $67.31 \%$ de nuestras pacientes tuvo el alumbramiento de 10 a 15 minutos no así en el $0.73 \%$ que se prolongó a 30 minutos, tiempo que está dentro de la media para un alumbramiento espontaneo, dentro del rango normal.

Del total de partos en posición vertical, el 100\% de los recién nacidos obtuvieron en la valoración del Apgar puntos variables entre 8-9 al minuto de vida, lo que refleja que en esta posición el expulsivo es menos traumante para el feto porque ha disminuido la compresión de la presentación sobre el suelo pélvico, es importante destacar que este dato no guarda relación con el de tinción del líquido amniótico que en el $10.34 \%$ estaba teñido. 
En relación a la perdida hemática, en el $48.28 \%$ de las mujeres fue inferior a $250 \mathrm{ml}$ y en el $18.5 \%$ de 300ml, cifras que están en el rango de lo normal si consideramos que la cantidad estimada es hasta 500ml en el parto por vía vaginal; nuestro hallazgo concuerda con los reportados por Calderón (2011), y discrepan por los reportados por Grupta (2013), quien considera que en la posición horizontal el sangrado del alumbramiento es menor con respecto a la posición vertical.

Fue posible constatar que del total de las 551 pacientes que optaron por la posición vertical para el parto, el $41.74 \%$ tuvieron edades entre 30 a 35 años, de los que el $81.49 \%$ fueron de raza indígena con instrucción primaria en el $45.37 \%$ del total de la muestra.

\section{Conclusiones}

- La mayor opción entre las parturientas fue por el parto en posición horizontal en contra de la posición vertical, probablemente este fenómeno ocurrió por falta de conocimiento o divulgación de las ventajas relacionados con el parto vertical.

- Se determinó que la duración del parto expulsivo en la posición vertical fue más breve al compararlo con la posición horizontal; esto guarda relación con la forma y dirección del canal del Parto, que en la posición vertical se presentan la modificación de los diámetros anteroposteriores y transversales de la pelvis, y la mujer está más motivada a ejercer el pujo de manera más efectiva.

- Existe libertad de elección, por parte de las mujeres para elegir la postura que más les agrada y la más confortable de acuerdo a sus costumbres para el momento del parto.

- Acerca del Apgar obtenido por los Recién Nacidos al minuto de vida, incluimos que el beneficio que aporta el Parto Vertical y que no se comprimen los grandes vasos que llevan sangre a la placenta y por ende la oxigenación al feto.

- Las prácticas obstétricas ancestrales, como parte de nuestra cultura, permiten minimizar las complicaciones del recién nacido Gineco -Obstetra.

- El parto en posición vertical en las instituciones de salud, ubicadas en áreas rurales, permite disminuir la mortalidad materna infantil, en dichas zonas donde la prevalencia de parto domiciliario aun es alta. 


\section{Referencias Bibliográficas}

Carrillo, (2016). Resultados perinatales de los partos atendidos en un hospital de tercer nivel de atención. Salud en Tabasco Vol. 22, No. 1 y 2, Enero-Abril, Mayo-Agosto 2016. Recuperado en https://tabasco.gob.mx/sites/default/files/users/ssaludtabasco/17_2.pd

Chávez (2007). Introducción a la Investigación Educativa. Maracaibo: Ars Gráfica.

EL UNIVERSO (2016). Noticias de Ecuador y el mundo en eluniverso.com. Revisa las principales noticias nacionales e internacionales: opinión, política, economía, internacional.

ENDEMAIN (2004). Encuesta Demográfica y de Salud Materna Infantil

Engelman (1997). En La gioia del parto: Segreti e virtù del corpo femminile durante il travaglio. Recuperado en Ehttps://books.google.com.ec/books?isbn=8886631790

Hernández, Fernández y Baptista, (2010). Metodología de la Investigación. McGraw Hill. México.

Martínez E; Martínez M; Toledo M; Pineda, J; Monleón J y Ferrero Pilar (2001). La depresión postparto: un estudio exploratorio con una muestra de mujeres valencianas. Revista de Psicopatología y Psicología Clínica, Vol. 6, núm. 1.

OMS (1985). Cuidados en el parto normal: una guía práctica (Informe presentado por el Grupo Técnico de Trabajo). Ginebra: Departamento de Salud Reproductiva.

Paniagua, J; Fernández, V (2013). ¿Es maltratada la mujer durante el parto?: Ensayo sobre la Humanización del Nacimiento. Ética de los Cuidados. 2013 jul-dic; 6(12). Recuperado de <http://www.index-f.com/eticuidado/n12/et9187.php> Consultado el 20 de mayo 2017.

Pozzio, María. (2014). El hecho de que sean más mujeres no garantiza nada: feminización y experiencias de las mujeres en la gineco-obstetricia en México. Salud Colectiva, v.10, n. 3, p. $325-337$.

Walker (2008). Cambio de la verticalidad a la horizontalidad para el parto. Recuperado de apsredes.org/wp-content/uploads/.../LAS-REDES-DE-ATENCION-DE-SALUD-web3.pd. 